\title{
Treacher Collins Syndrome : A Case Report
}

Tarannum Morshed ${ }^{1 *}$

Fonindro Nath Paul ${ }^{2}$

R N Sarker ${ }^{2}$

Shahara Haque ${ }^{2}$

Sadia Sultana ${ }^{2}$

'Department of Radiology \& Imaging Bangladesh Institute for Research \& Rehabilitation in Diabetes, Endocrine \& Matabilic Diseases (BIRDEM) Dhaka, Bangladesh.

${ }^{2}$ Department of Radiology \& Imaging Dhaka Medical College

Dhaka, Bangladesh.

\section{*Correspondence to:}

\section{Dr. Tarannum Morshed}

Senior Medical Officer Department of Radiology \& Imaging Bangladesh Institute for Research \& Rehabilitation in Diabetes,

Endocrine \& Matabilic Diseases (BIRDEM) Dhaka, Bangladesh.

Mobile : +8801818464627

E-mail: awwal70@yahoo.com

\begin{abstract}
Treacher Collins syndrome (TCS) or Franceschetti syndrome is an autosomal dominant disorder of craniofacial development with variable expressivity. It is named after Edward Treacher Collins (1862-1932), the English surgeon and ophthalmologist, who described the essential components of the condition in 1900 . Incidence of this syndrome is approximately 1 in 50,000 live births and it affects both genders equally. The typical physical features include downward slanting eyes, micrognathia (a small lower jaw), Conducting hearing loss, under developed zygoma, drooping part of lateral lower eyelids, and malformed or absent ears. This article describes clinical and radiological features of TCS in a $07 \mathrm{yr}$ old female child who had reported to the department of Dentistry and Radiology with the complaint of forwardly placed upper anterior teeth and hearing loss. Also pathogenesis, prenatal diagnosis, differential diagnosis, management and preventive aspects are discussed.
\end{abstract}

Key words : Treacher collins syndrome (TCS); zygoma; micrognathia.

\section{INTRODUCTION}

Treacher Collins syndrome is a severe congenital disorder of craniofacial development characterized by numerous developmental anomalies that are restricted to the head and face. Growth of craniofacial structures derived from the first and second pharyngeal arch, groove, and pouch is diminished symmetrically and bilaterally ${ }^{1}$. Hypoplasia of the facial bones, particularly the mandible and zygomatic complex, is an extremely common feature of TCS. Although the condition was probably first described by Thomson (1846) followed by Toynbee (1847) and Berry (1889), it is named after E Treacher Collins who described the essential components of the syndrome in 1900. The first extensive review of the condition was done by Franceschetti and Klein in 1949, who coined the term mandibulofacial dysostosis to describe the clinical features ${ }^{2}(1$ 1. Dixon MJ. Treacher Collins syndrome. J Med Genet 1995; 32: 806-8. ). This a rare genetic disorder that causes abnormal development of facial bones and tissues, resulting in a wide range of facial defects. Symptoms vary from barely noticeable to severe. In general, most people have underdeveloped facial bones, especially cheekbones. Hearing loss is a common complication of TCS because some people have malformed ears. In very severe cases, underdeveloped facial bones may make it difficult for the patient to breathe, eat, or both ${ }^{3}$.

\section{CASE REPORT}

A 7 years old female child (Fig.1) Presented to the dept. of Dentistry with facial deformity, difficulty in eating, deafness and growth retardation. Her parents were nonconsanguinous and had no significant family history. Past history revealed she was delivered at home at term. She was not breast fed due to difficulty in swallowing and still has complaints of dribbling of liquids from her mouth. Parents could not give proper immunization history. She had microtia. There were multiple facial dysraphism including downward- slanting eyes, malar hypolasia, mandibular hypoplasia a9mirognathia) and a large mouth.

Her x-ray skull and mandible lateral view revealed mandible is hypoplastic, significantly affecting the ramus (fig.2). Facial bones appears hypoplastic. X-ray mastoid (town's view) was reported as paucity of air cells in mastoid region with hypoplastic petrous part of temporal bone.

CT scan of maxilla-facial region had following findings 
Face

- All the facial bones are hypoplastic.

- Zygomatic arch is not developed.

- Body of mandible is hypoplastic and significantly affecting the ramus.

Ear

- External auditory canal and tympanic cavity are dysmorphic (congenitally deficient).

- Non visualization of mastois air cells (congenitally deficient).

\section{Palate}

- Hard palate is hypoplastic.

Final diagnosis was- features more in favor of Treacher Collins syndrome.
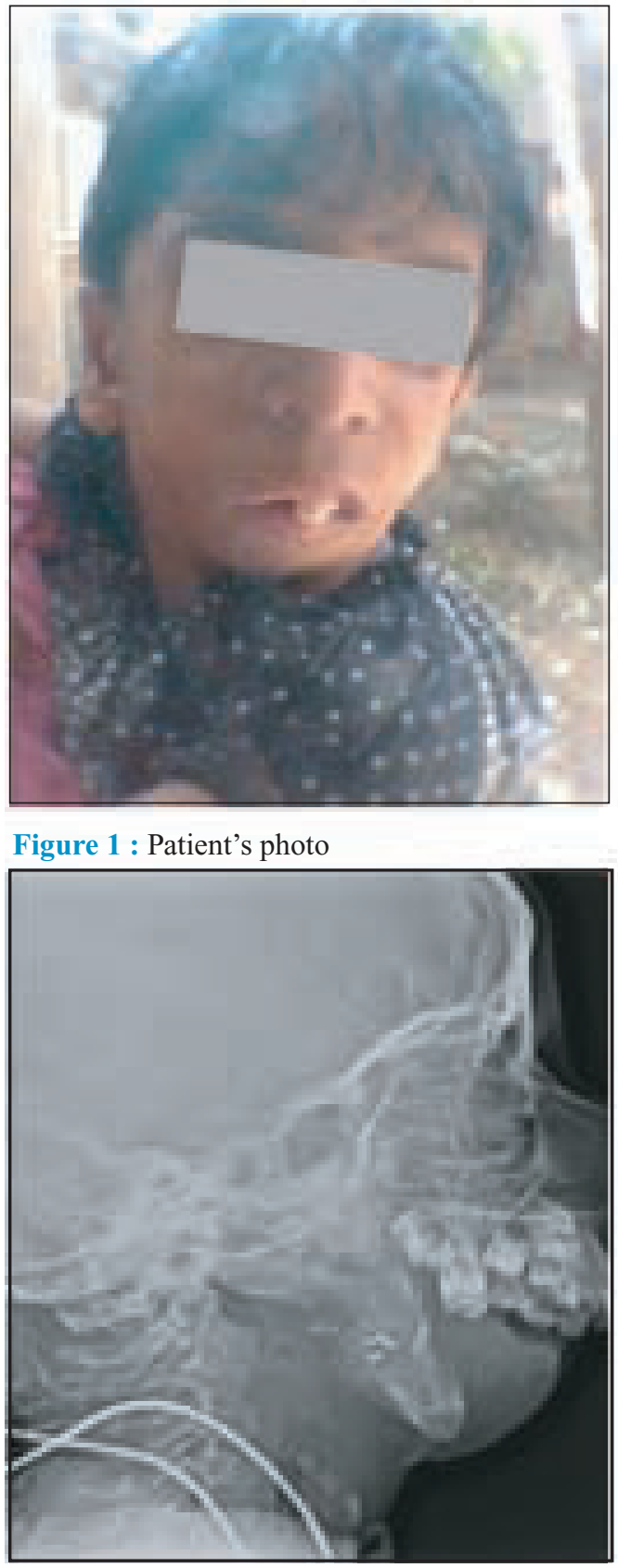

Figure 2 : Hypoplastic mandible

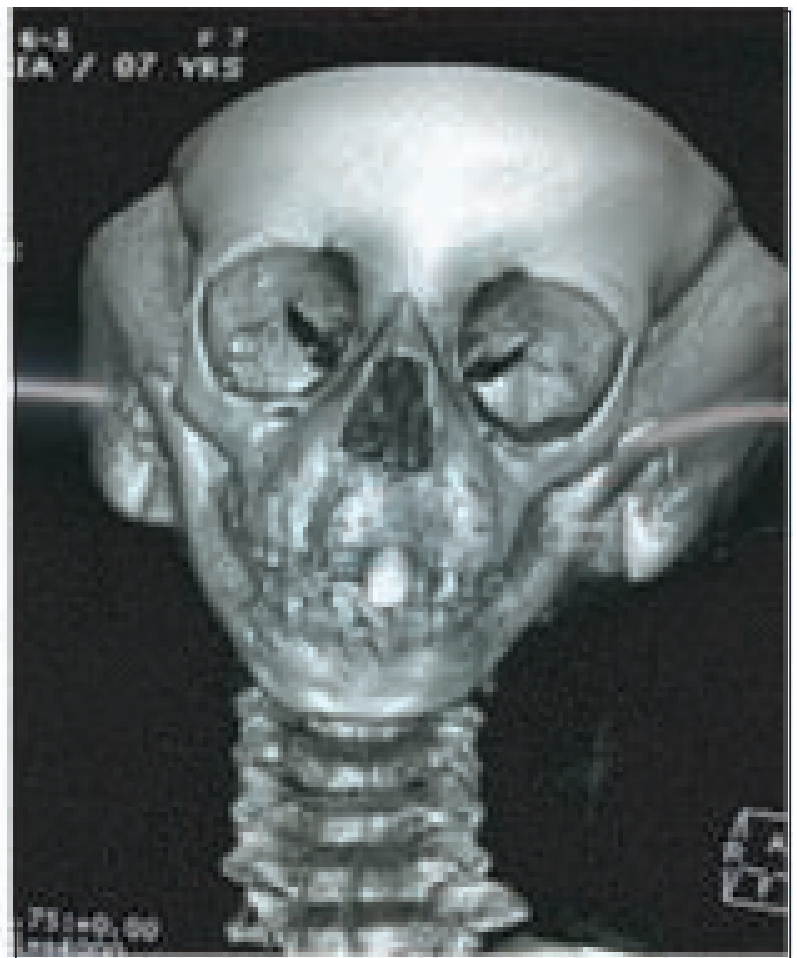

Figure 3 : CT scan of face (3D reconstruction) showing hypoplastic facial bones and mandible

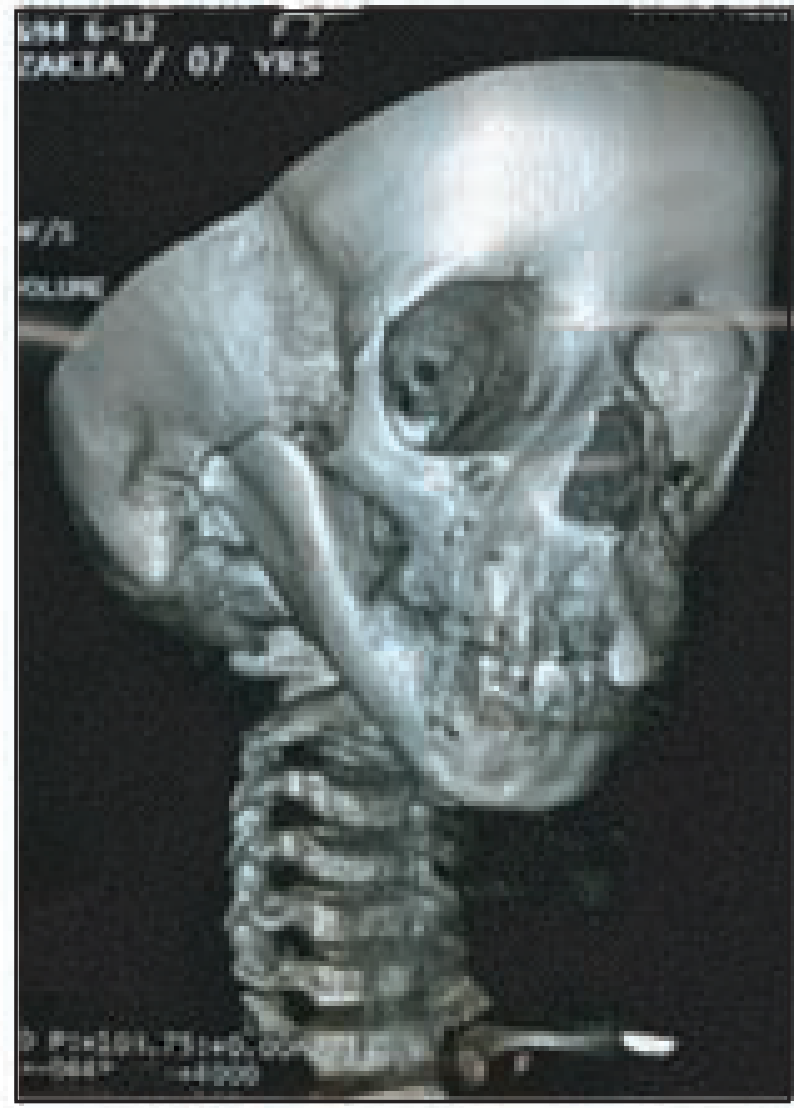

Fig;ure 4 : CT scan of face (3D reconstruction) showing hypoplasia of mandibuar ramus and absence of zygomatic arch. 


\section{DISCUSSION}

Treacher Collins syndrome or Franceschetti syndrome is an autosomal dominant disorder of craniofacial development which has an incidence of approximately 1 in 50,000 live births $^{3}$. It affects both genders equally. While $40 \%$ of cases have a family history, the remaining $60 \%$ appear to arise as a result of a de novo mutation ${ }^{4,5}$.

Several hypotheses were proposed to explain the pathogenesis of TCS including abnormal patterns of neural crest cell migration, abnormal domains of cell death, improper cellular differentiation during development or an abnormality of the extracellular matrix ${ }^{6}$. Growth of craniofacial structures derived from the first and second pharyngeal arch, groove, and pouch is diminished symmetrically and bilaterally. The condition is recognizable at birth and can also be diagnosed prenatally based on ultrasonography findings?

Recently genetic, physical and transcript mapping techniques have identified the gene mutated in TCS which is designated asTCOF1 and mapped to human chromosome 5q32-33.2locus. It was found to encode a low complexity, serine/alanine-rich, nucleolar phosphoprotein termed Treacle ${ }^{8}$. Valdez et al. suggested that haploinsufficiency of treacle in TCS patients might cause insufficient rRNA production in the prefusion neural folds, resulting in abnormal craniofacial development. (The cephalic neural crest cells probably require a higher threshold concentration of rRNA for their survival and proper differentiation during early embryogenesis 9 .

Clinical features of Treacher Collins syndrome ${ }^{1}$.

The presentation of symptoms in people with Treacher Collins Syndrome varies. Some individuals may be so mildly affected that they remain undiagnosed, others can have severe facial involvement and life-threatening airway compromise. Most of the features of TCS are bilateral and are already recognizable at birth. The most life-threatening problem of individuals with TCS is a constricted airway, since this can give problems with breathing.

Face

- The nose has a normal size; however, it appears large because of hypoplastic supraorbital rims and hypoplastic zygomas.

- The palpebral fissures are downward-sloping, the cheekbones are depressed, the pinnae are malformed with widely varying severity, and the chin recedes with a large, down-turned mouth.

Skull

- On radiographs, the malar bones, zygomatic process of frontal bone, lateral pterygoid plates, paranasal sinuses, and mandibular condyles are hypoplastic.

- The mastoids are not pneumatized.

- The lateral margins of the orbits may be defective, and the orbits are hyperteloric.

- The cranial base is progressively kyphotic.

- The calvaria are essentially normal.
Eyes

- The palpebral fissures are short and slope laterally downward.

- In the outer third of the lower lid, a coloboma is present, and the cilia (i.e., eyelashes) may be deficient medially from the lower lid, as is seen in the image below.

Ears

- The pinnae are often malformed, crumpled forward, or misplaced toward the angle of the mandible, as is seen in the image below

- Frequently, meatal atresia, external auditory canal stenosis or atresia, hypoplasia or agenesis of the malleus and the incus, monopodal stapes, ankylosis of stapes in the oval window, and absence of the middle ear and tympanic spaces are present, resulting in a conductive hearing loss.

- The inner ears are normal.

- Extra ear tags and blind fistulas may develop anywhere between the tragus and the angle of the mouth.

Nose

- The nose appears large because of the lack of malar development and hypoplastic supraorbital ridges. Mouth and throat

- A cleft palate is found in one third of patients with Treacher Collins syndrome, and congenital palatopharyngeal incompetence (foreshortened, immobile, or absent soft palate; submucous cleft palate) is found in an additional one third of patients.

- The parotid glands are missing or hypoplastic.

- Pharyngeal hypoplasia is a constant finding.

- Radiographically, the mandibular angle is more obtuse than normal and the ramus is deficient. The coronoid and condyloid processes are flat or aplastic.

Mental status

- Intelligence is usually normal.

- Developmental delay may be secondary to undiagnosed hearing loss.

Dysfunctional symptoms

- Hypoplasia and a retropositioned tongue

- Difficulties with swallowing and feeding (caused by musculoskeletal underdevelopment and a cleft palate)

- Conductive hearing loss (caused by maldevelopment of the auditory canal and middle ear ossicles)

- Impaired vision (caused by underdeveloped lateral orbit and extraocular muscle

\section{Radiological findings}

The anomalies can be studied using conventional radiological procedures or multislice CT scan with three-dimensional reconstruction. The imaging characteristics include hypoplastic or absent zygomatic arches - whereas the body of the zygoma itself is the least affected part, choanal shortening, a small mandible with retrognathism, and narrowing of the maxilla. The CT findings of the temporal bone consist of a malformation of the external and middle ear with an underdeveloped temporal bone and absent or reduced mastoid pneumatization. 
The inner ear structures are normal since they are not derived from the branchial arches but from the epidermal otic placode. The bony external auditory canal is absent or hypoplastic. The middle ear malformation includes a hypoplastic and dysmorphic middle ear space with dysmorphic or missing ossicles and with ossicular disjunction. In most cases, a soft tissue density fills the tympanic cavity. A common finding is an increased angle of the genu of the facial nerve

(normally between 60 to $80^{\circ}$ ), with the facial nerve running more laterally and dysplasia of the stapesfacial nerve complex that often makes the middle ear malformation uncorrectable ${ }^{11}$. Treatment of MFD consists of surgical repair of the anomalous facial development and a bone conducting hearing aid.

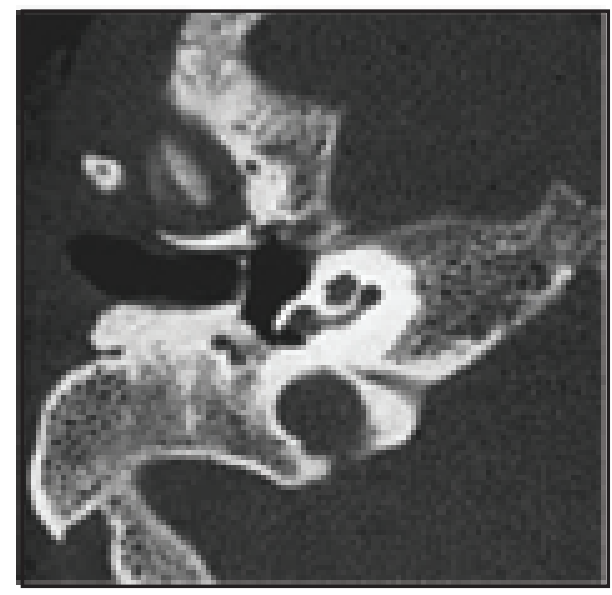

Figure 5 a

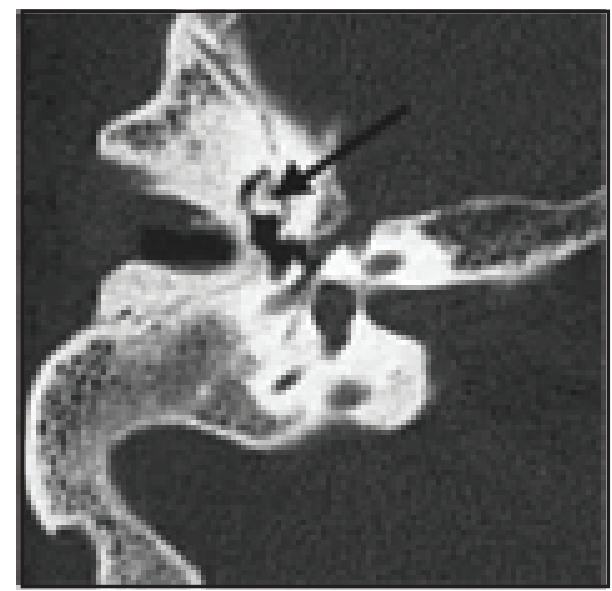

Figure $5 \mathrm{~b}$

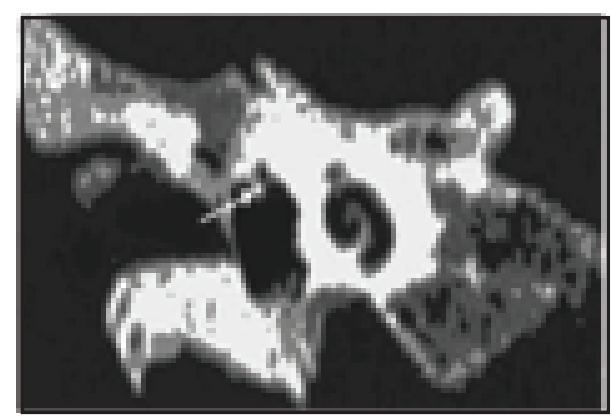

Figure 5 c
A high resolution multidetector helical CT scan of the temporal bones was performed, with a collimation of 0.75 $\mathrm{mm}$. On axial CT image (Fig. $5 \mathrm{a}, \mathrm{b}$ ) and coronal reformatted image (c) through the right middle ear., there is a reduced mastoid pneumatization and a malformation of the middle ear with a dys-/hypoplastic malleus and incus (arrow) and an absent stapes.

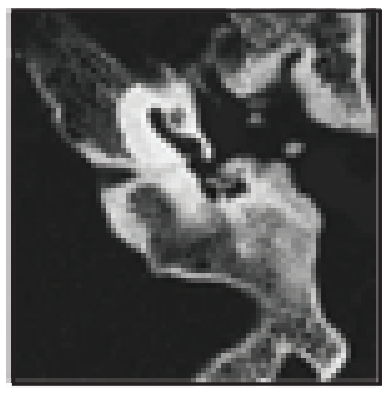

Figure 6 a

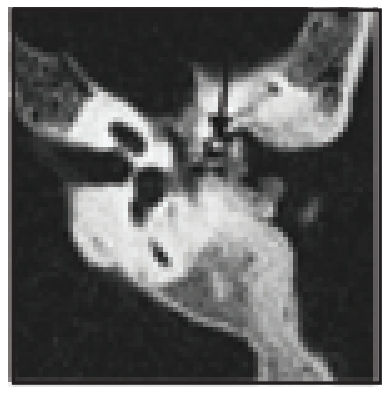

Figure 6 b

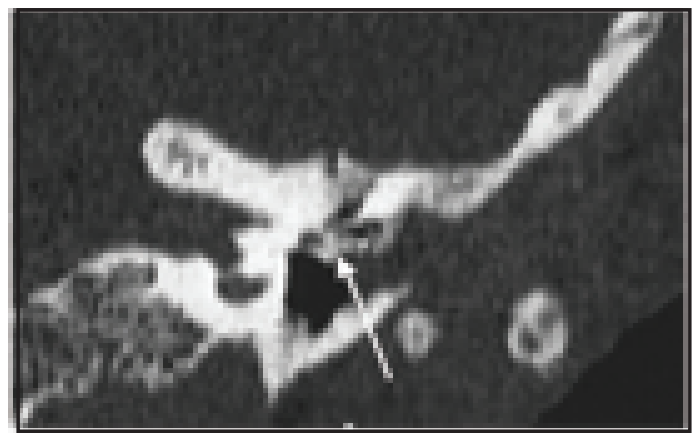

Figure $6 \mathrm{c}$

On axial CT image (Fig. 2 a,b) and coronal reformatted image (c) through the left middle ear, there is a partial agenesis of the external auditory canal, absent mastoid pneumatization with only partial pneumatization of the middle ear and hypoplastic ossicles (arrow

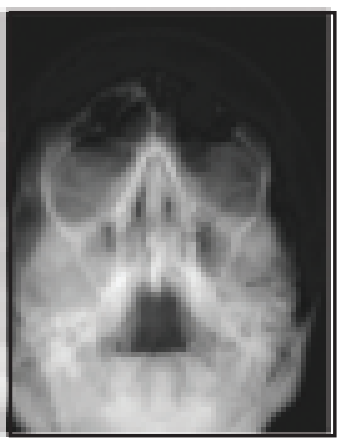

Figure 7 : X-Ray: hypoplasia of zygomatic bone.

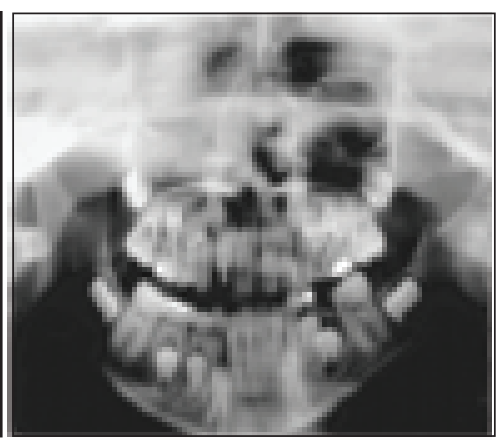
process, mandibular hypoplasia and hypoplasia of mandibular ramus
Figure 8 : OPG-agenesis of coronoind

Treatment for Treacher Collins syndrome (TCS) depends on your child's age and how much they are affected. Because your child's face grows along with your child, the timing of the treatments is very important. The treatment of individuals affected by TCS needs a multidisciplinary approach and may involve the intervention of different professionals.

If there is a cleft palate, the repair normally takes place at the age of 9-12 months. 
The hearing loss is treated by bone conduction amplification, speech therapy and educational intervention to avoid language/speech problems. The bone-anchored hearing aid (Baha) is an alternative for individuals with ear anomalies.

The zygomatic and orbital reconstruction is performed when the cranio-orbitozygomatic bone is completely developed. Usually at the age of 5-7 years. In children an autologous bone graft is mostly used.

- The optimal age for the maxillomandibular reconstruction is still a major point of discussion, at present, this classification is generally used:

1. Type I (mild) and Type IIa (moderate) 13-16 years

2. Type IIb (moderate to severe malformation) at skeletal maturity

3. Type III (severe) 6-10 years
Hearing loss in Treacher Collins Syndrome is caused by deformed structures in the outer and middle ear. The hearing loss is generally bilateral. Attempts to surgically reconstruct the external auditory canal and improve hearing in children with Treacher Collins Syndrome have not yielded positive results. Auditory rehabilitation with BAHA or a conventional bone conduction aid has proven preferable to surgical reconstruction $^{12,13}$.

\section{CONCLUSION}

Radiology plays key role in the diagnosis and treatment of Treacher Collins syndrome. Individuals with severe form of TCS usually undergo, over a period of time, multiple major reconstructive surgeries that are rarely fully corrective and stem cell therapy is unlikely to benefit the reconstructive repair of severe craniofacial malformations. Consequently, more research should be directed on preventive aspects of this syndrome.

\section{DISCLOSURE}

All the authors declared no competing interest.

\section{REFERENCES}

1. Marie MT. Mandibulofacial Dysostosis (Treacher Collins Syndrome) Med scape march 9, 2012.

2. Dixon MJ. Treacher Collins syndrome. J Med Genet 1995; 32: 806-808.

3. Crovetto $\mathrm{MA}^{1}$, Linthicum FH. Treacher Collins syndrome (mandibulofacial dysostosis). Jr.Otol Neurotol. 2002; Nov;23(6):1005.

4. Dixon MJ, Read AP, Donnai D, Colley A, Dixon J, Williamson R. The Gene for Treacher Collins Syndrome Maps to the Long Arm of Chromosome 5. Am J Hum Genet 1991; 49: 17-22.

5. Edwards S, Gladwin A, Dixon M. The Mutational spectrum In Treacher Collins Syndrome reveals a predominance of Mutations that Create a Premature-Termination Codon. Am J Hum Genet ;1997; 60: 515-524.

6. Treacher Collins syndrome- a case report and review of literature. Vikrant Kasat 1, Rahul Baldawa J Clin Exp Dent. 2011;3(Supp11):e395-399.

7. [Guideline] Cunniff C. Prenatal screening and diagnosis for pediatricians. Pediatrics. Sep 2004;114(3):889-894. [Medline].

8. Trainor P, Dixon J, Dixon M. Treacher Collins syndrome: etiology, pathogenesis and prevention. Eur J Hum Genet 2009; 17: 275-283.

9. Valdez B, Henning D, So R, Dixon J, Dixon M. The Treacher Collins syndrome (TCOF1) gene product is involved in ribosomal DNA gene transcription by interacting with upstream binding factor. Proc Natl Acad Sci USA 2004; 101:10709-714

10. C. Geniets, Ö. Özsarlak, M. Maes, J.W. Van Goethem, P.M. Parizel. TREACHER-COLLINS SYNDROME ;JBR-BTR, 2006; 89: 132-133.

11. C. Geniets, Ö. Özsarlak, M. Maes, J.W. Van Goethem, P.M. Parizel. TREACHER-COLLINS SYNDROME ;JBR-BTR, 2006, 89: 132-133. Fig. 5 \& 6 A high resolution multidetector helical CT scan of the temporal bones

12. Ribeirão PB. Goldenher syndrome- acase report.Dent. J. vol.14 no.1 June 2003. Fig.8 Mandibular hypoplasia

13. Wikipedia,the free encyclopedia. Treacher Collins Syndrome [document on the internet] [updated 2012 Nov 8]. Available from: file://1:Treacher Collins Syndrome-Wikipedia, the free encyclopedia. 